\title{
Chapter 7 \\ Heterogeneous Dynamic Stress Drops on Asperities in Inland Earthquakes Caused by Very Long Faults and Their Application to the Strong Ground Motion Prediction
}

\author{
Kazuo Dan, Masanobu Tohdo, Atsuko Oana, Toru Ishii, \\ Hiroyuki Fujiwara, and Nobuyuki Morikawa
}

\begin{abstract}
We compiled the stress drops on the asperities in inland earthquakes caused by strike-slip faults. Then, we applied the log-normal distribution to the data and obtained the medium of $10.7 \mathrm{MPa}$ and the logarithmic standard deviation of 0.45. Also, we compiled the stress drops on the asperities in inland earthquakes caused by reverse faults and obtained the medium of $17.1 \mathrm{MPa}$ and the logarithmic standard deviation of 0.39 .

By using the obtained log-normal distributions, we examined a procedure for assigning the heterogeneous dynamic stress drops to each asperity. We adopted 12.2 MPa, which had been estimated by Dan et al. (J Struct Constr Eng (Trans Archit Inst Japan), 76:(670):2041-2050, 2011) for long strike-slip faults, as the medium, and 18.7 MPa, which had been estimated by Dan et al. (J Struct Constr Eng (Trans Archit Inst Japan), 80(707):47-57, 2015) for long reverse faults.

Moreover, we truncated the log-normal distributions of the dynamic stress drops on the asperities at the value of $3.4 \mathrm{MPa}$ for strike-slip faults and of $2.4 \mathrm{MPa}$ for reverse faults because they should be larger than the dynamic stress drop averaged over the entire fault.

Finally, we proposed a procedure for evaluating fault parameters taking into account of the heterogeneous dynamic stress drops on the asperities and calculated strong ground motions. The results had wider variations of the peak ground accelerations and velocities than those with uniform dynamic stress drops on the asperities, while the averages were almost the same.
\end{abstract}

\author{
K. Dan $(\bowtie) \cdot$ A. Oana $\bullet$ T. Ishii \\ Institute of Technology, Shimizu Corporation, Tokyo, Japan \\ e-mail:kazuo.dan@shimz.co.jp \\ M. Tohdo \\ Ohsaki Research Institute, Inc., Chiyoda-ku, Tokyo, Japan \\ H. Fujiwara • N. Morikawa \\ National Research Institute for Earth Science and Disaster Prevention, Tokyo, Japan
}

K. Kamae (ed.), Earthquakes, Tsunamis and Nuclear Risks,

DOI 10.1007/978-4-431-55822-4_7 
Keywords Strong motion prediction - Inland earthquake $\bullet$ Long fault $\bullet$ Fault parameter $\bullet$ Asperity $\bullet$ Dynamic stress drop $\bullet$ Heterogeneity

\subsection{Introduction}

Dan et al. [1, 2] proposed a procedure for evaluating the parameters of long strikeslip faults, evaluated fault parameters based on the proposed procedure, and calculated strong ground motions. Also, Dan et al. [3] carried out the same study for long reverse faults. In these studies, they treated the dynamic stress drops on the asperities as the uniform ones. But, it is hard to assume that all the dynamic stress drops on the asperities would be uniform in the actual earthquakes. Especially, in long faults, the number of the asperities is thought to be large, and the heterogeneity of the dynamic stress drops is easier to be observed. This tendency should cause large effects on the spatial distribution of the predicted strong motions.

Hence, in this paper, we proposed a procedure for evaluating fault parameters taking into account the heterogeneous dynamic stress drops on the asperities and calculated strong ground motions to compare the results with ground motion prediction equations by $\mathrm{Si}$ and Midorikawa [4] and with the results with uniform dynamic stress drops on the asperities.

\subsection{Statistics of the Heterogeneous Stress Drops on the Asperities}

\subsubsection{Strike-Slip Faults}

At first, we compiled heterogeneous stress drops on the asperities in the past inland earthquakes caused by strike-slip faults. Table 7.1 shows stress drops on the asperities or SMGAs (strong motion generation areas) in the past earthquakes obtained by previous studies [5-9]. In Table 7.1, when the stress drops on the asperities in one earthquake were different from each other, each value was adopted independently, but when all the values of the stress drops on the asperities in one earthquake

Table 7.1 Stress drops on the asperities in the past inland earthquakes caused by strike-slip faults

\begin{tabular}{l|l|l|l|l}
\hline \multirow{2}{*}{ Earthquakes } & & \multicolumn{3}{l}{ Stress drops on the asperities (MPa) } \\
\cline { 5 - 5 } & References & $\Delta \sigma_{\text {asp1 }}$ & $\Delta \sigma_{\text {asp2 }}$ & $\Delta \sigma_{\text {asp3 }}$ \\
\hline 1995 Hyogo-Ken Nanbu & Kamae and Irikura [5] & 8.6 & 16.3 & 8.6 \\
\hline 1999 Kocaeli, Turkey & Kamae and Irikura [6] & 12.0 & 5.0 & 10.0 \\
\hline 2000 Tottori-ken Seibu & Ikeda et al. [7] & 28.0 & 14.0 & - \\
\hline$\prime \prime$ & Muto et al. [8] & 8.7 & 7.3 & - \\
\hline 2005 Fukuoka-ken Seiho-oki & Satoh and Kawase [9] & 11.3 & 11.3 & - \\
\hline
\end{tabular}


Fig. 7.1 Fitting of the log-normal distribution to the stress drops on the asperities in the past inland earthquakes caused by strike-slip faults

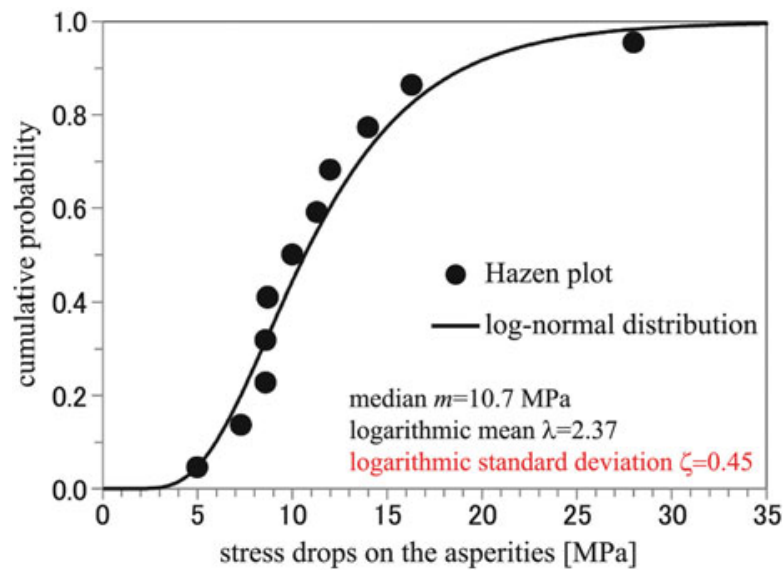

were the same, that value was adopted as one data. Here, the stress drop $\Delta \sigma$, also called static stress drop, is the difference between the initial shear stress on the fault before the earthquake and final shear stress after the earthquake at the time all the rupture on the fault terminates and the stress status becomes stable, and the dynamic stress drop $\Delta \sigma^{\#}$ is the difference between the initial shear stress and the shear stress at the time the rupture terminates at a certain point on the fault while the rupture may not terminate at other points. Although the stress drop $\Delta \sigma$ and the dynamic stress drop $\Delta \sigma^{\#}$ are different from each other in general, we assumed the difference to be negligible in this paper.

When the number of the stress drops was $K$, we adopted Hazen plot and assigned a cumulative probability (non-exceeding probability) $P_{k}$ as follows:

$$
P_{k}=1-\frac{k-0.5}{K}
$$

We calculated a logarithmic mean and a logarithmic standard deviation of the data and fitted a log-normal distribution to the data. Figure 7.1 shows the result. The logarithmic mean of the stress drops was calculated to be 2.37 (the median $=10.7 \mathrm{MPa}$ ) and the logarithmic standard deviation to be 0.45 .

\subsubsection{Reverse Faults}

Next, we compiled heterogeneous stress drops on the asperities in the past inland earthquakes caused by reverse faults.

Table 7.2 shows stress drops on the asperities or SMGAs (strong motion generation areas) in the past earthquakes obtained by previous studies $[6,10-18]$.

We calculated a logarithmic mean and a logarithmic standard deviation of the data and fitted a log-normal distribution to the data. Figure 7.2 shows the result. 
Table 7.2 Stress drops on the asperities in the past inland earthquakes caused by reverse faults

\begin{tabular}{l|l|l|l|l|l}
\hline \multirow{2}{*}{ Earthquakes } & \multirow{4}{*}{ References } & \multicolumn{4}{|c}{ Stress drops on the asperities (MPa) } \\
\cline { 3 - 6 } & & $\Delta \sigma_{\text {asp1 }}$ & $\Delta \sigma_{\text {asp2 }}$ & $\Delta \sigma_{\text {asp3 }}$ & $\Delta \sigma_{\text {asp4 }}$ \\
\hline 1999 Chi-Chi, Taiwan & Kamae and Irikura [6] & 10.0 & 10.0 & 10.0 & - \\
\hline 2004 Niigata-Chuetsu & Kamae et al. [10] & 7.0 & 20.0 & - & - \\
\hline$\prime \prime$ & Satoh et al. [11] & 26.7 & 13.4 & - & - \\
\hline 2007 Noto-Hanto & Kamae et al. [12] & 20.0 & 20.0 & 10.0 & - \\
\hline$\prime \prime$ & Kurahasi et al. [13] & 25.8 & 10.3 & - & - \\
\hline 2007 Niigata-Chuetsu-oki & Irikura et al. [14] & 23.7 & 23.7 & 19.8 & - \\
\hline$\prime \prime$ & Kamae and Kawabe [15] & 18.4 & 27.6 & 27.6 & - \\
\hline 2008 Iwate-Miyagi Nairiku & Kamae [16] & 13.8 & 13.8 & - & - \\
\hline$\prime \prime$ & Irikura and Kurahashi [17] & 17.0 & 18.5 & - & - \\
\hline 2008 Wenchuan, China & Irikura and Kurahashi [18] & 13.2 & 13.2 & 13.2 & 13.2 \\
\hline
\end{tabular}

Fig. 7.2 Fitting of the log-normal distribution to the stress drops on the asperities in the past inland earthquakes caused by reverse faults

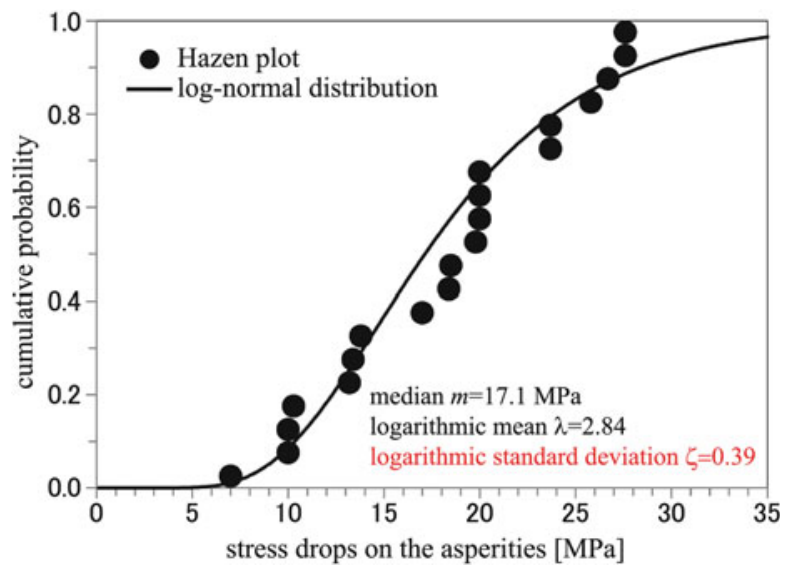

The logarithmic mean of the stress drops was calculated to be 2.84 (the median $=17.1 \mathrm{MPa}$ ) and the logarithmic standard deviation to be 0.39 .

\subsection{Procedure for Evaluating Fault Parameters}

We examined how to assign the heterogeneous stress drops to each asperity based on the cumulative probability distribution obtained in Sect. 7.2 for the strong motion prediction.

The median of the stress drops on the asperities in strike-slip faults is consistent with the value of 12.2 MPa estimated by Dan et al. [1] as the geometrical mean of the dynamic stress drops on the asperities in strike-slip faults and that for reverse 
faults is consistent with the value of $18.7 \mathrm{MPa}$ estimated by Dan et al. [3] as the geometrical mean of the dynamic stress drops on asperities in reverse faults. Hence, we adopted 12.2 MPa as the median for strike-slip faults and 18.7 MPa for reverse faults. As for the variation, we adopted 0.45 as the logarithmic standard deviations for strike-slip faults as shown in Fig. 7.1 and 0.39 for reverse faults as shown in Fig. 7.2.

Figure 7.3 shows the cumulative probability function for strike-slip faults indicated by the red line. Here, we truncated the function less than $3.4 \mathrm{MPa}$ because the averaged dynamic stress drop on the entire fault is $3.4 \mathrm{MPa}$ [1]. For reverse faults, we truncated the function less than $2.4 \mathrm{MPa}$ because the averaged dynamic stress drop on the entire fault is $2.4 \mathrm{MPa}$ [3].

We chose the stress drop at the middle point in the line divided equally of the vertical axis for the cumulative probability function, as shown in Fig. 7.3 based on the idea of Hazen plot, and assigned it to the dynamic stress drop on each asperity.

When we apply the heterogeneity to the dynamic stress drops on the asperities, the seismic moment and the short-period level would become different from those of the original fault model with the uniform dynamic stress drops on the asperities. Here, the short-period level is the flat level of the acceleration source spectrum in the short-period range. Also, the relationship would not be preserved that the slips on the asperities should be proportional to the dynamic stress drops on the asperities and the equivalent radii of the asperities if the slips on the asperities obey the similar trend as the equation of the constant stress drop on a circular crack. Hence, we preserved the seismic moment by adjusting the areas of the asperities so that the averaged dynamic stress drop on the entire fault should be preserved. Also, we reevaluated the slips on the asperities so that the relationship was preserved that the slips on the asperities should be proportional to the dynamic stress drops on the asperities and the equivalent radii of the asperities.

Because it is impossible to preserve the short-period level, we just confirmed that the value of the short-period level did not change largely. In this paper, we assigned the heterogeneous dynamic stress drops to the asperities randomly.

Fig. 7.3 Generated examples of the heterogeneous dynamic stress drops on the asperities in the strike-slip fault $(N=21)$

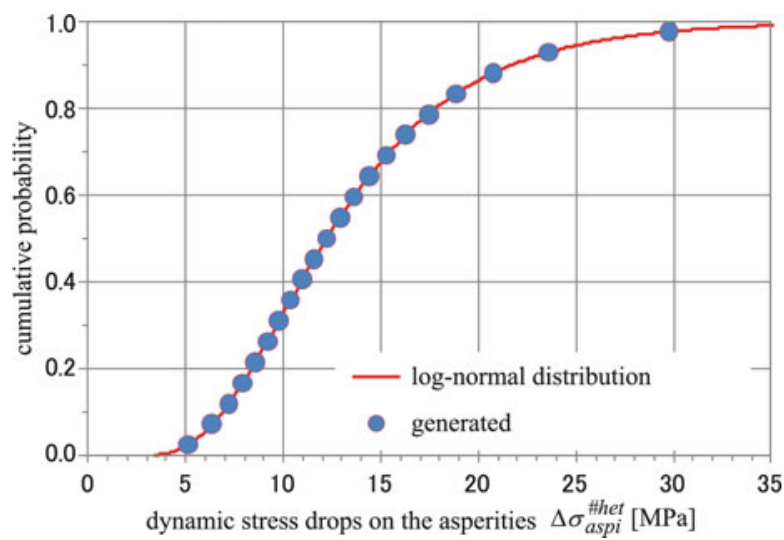


When we evaluate fault parameters with heterogeneous dynamic stress drops, we first assume the parameters with the uniform dynamic stress drop such as the areas $S_{\text {aspi }}$ of the $i$-th asperity, the dynamic stress drop $\Delta \sigma_{\text {asp }}^{\#}$, and the averaged slip $D_{\text {asp }}$ on the asperities.

We put the heterogeneous stress drop on the asperity as $\Delta \sigma_{\text {aspi }}^{\# \text { het which is }}$ generated by the way of Fig. 7.3. When we write the area of the $i$-th asperity with heterogeneous dynamic stress drop as $S_{\text {aspi }}^{\text {het }}$ and its ratio to $S_{\text {aspi }}$ as $p$ as follows:

$$
S_{\text {aspi }}^{\text {het }}=p S_{\text {aspi }}
$$

we obtain

$$
\begin{aligned}
\left(\sum_{i} \Delta \sigma_{\text {aspi }}^{\# \text { het }} S_{\text {aspi }}^{\text {het }}\right) / S_{\text {seis }} & =\left(p \sum_{i} \Delta \sigma_{\text {aspi }}^{\# \text { het }} S_{\text {aspi }}\right) / S_{\text {seis }} \\
& =\left(\sum_{i} \Delta \sigma_{\text {asp }}^{\#} S_{\text {aspi }}\right) / S_{\text {seis }}
\end{aligned}
$$

because the averaged dynamic stress drop on the entire fault $S_{\text {seis }}$ should be preserved.

Then, we obtain

$$
p=\left(\sum_{i} \Delta \sigma_{\text {asp }}^{\#} S_{\text {aspi }}\right) /\left(\sum_{i} \Delta \sigma_{\text {aspi }}^{\# \text { het }} S_{\text {aspi }}\right)
$$

The $S_{\mathrm{aspi}}^{\text {het }}$ can be evaluated by substituting $p$ of Eq. (7.4) for Eq. (7.2).

On the other hand, the slip $D_{\text {aspi }}^{\text {het }}$ on the asperity should be proportional to the dynamic stress drop on the asperity and the equivalent radius of the asperity. Hence, if we write the proportionality constant as $q$, the $D_{\text {aspi }}^{\text {het }}$ can be written by

$$
D_{\text {aspi }}^{\text {het }}=q \Delta \sigma_{\text {aspi }}^{\# \text { het }} \sqrt{S_{\text {aspi }}^{\text {het }} / \pi} \text {. }
$$

The averaged slip $D_{\text {asp }}$ on the asperities is obtained by

$$
\begin{aligned}
D_{\text {asp }} & =\left(\sum_{i} D_{\text {aspi }}^{\text {het }} S_{\text {aspi }}^{\text {het }}\right) / \sum_{i} S_{\text {aspi }}^{\text {het }} \\
& =\left(q \sum_{i} \Delta \sigma_{\text {aspi }}^{\text {\#het }} S_{\text {aspi }}^{\text {het }} \sqrt{S_{\text {aspi }}^{\text {het }} / \pi}\right) / \sum_{i} S_{\text {aspi }}^{\text {het }}
\end{aligned}
$$

Then, we obtain 
7 Heterogeneous Dynamic Stress Drops on Asperities in Inland Earthquakes...

$$
q=D_{\text {asp }} \sum_{i} S_{\text {aspi }}^{\text {het }} /\left(\sum_{i} \Delta \sigma_{\text {aspi }}^{\# \text { het }} S_{\text {aspi }}^{\text {het }} \sqrt{S_{\text {aspi }}^{\text {het }} / \pi}\right) .
$$

The $D_{\text {aspi }}^{\text {het }}$ can be evaluated by substituting $q$ of Eq. (7.7) for Eq. (7.5). In addition, in the case that the $D_{\text {aspi }}^{\text {het }}$ is not larger than the averaged slip $D$ on the entire fault, we should assign again the heterogeneous dynamic stress drops to the asperities randomly.

It is also necessary to evaluate the parameters for the background, and we can adopt the same procedure as those by Dan et al. [1] and Dan et al. [3] for evaluating the parameters of the fault model with the uniform dynamic stress drops on the asperities.

Figure 7.4 shows the evaluation procedure of the fault parameters mentioned above.

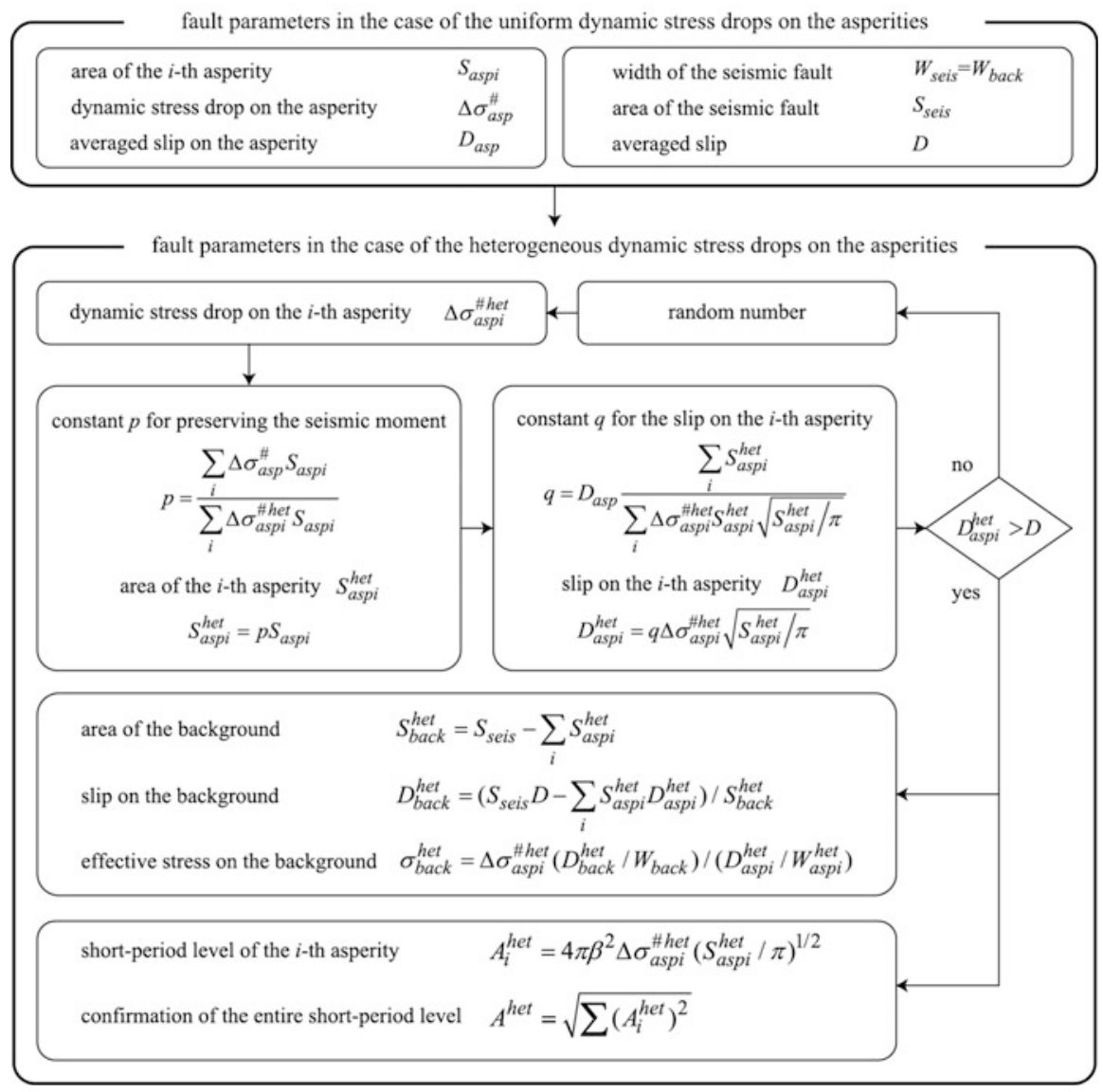

Fig. 7.4 Evaluation procedure of the fault parameters in the case of the heterogeneous dynamic stress drops on the asperities 


\subsection{Examples of Strong Motion Prediction Under Heterogeneous Dynamic Stress Drops on the Asperities}

For the active strike-slip fault $360 \mathrm{~km}$ long along the Median Tectonic Line, Japan, shown in Fig. 7.5, we made two models with the uniform dynamic stress drops on the asperities and with the heterogeneous dynamic stress drops.

Figure 7.6 shows the fault model with the uniform dynamic stress drops on the 21 asperities. Figure 7.7 shows the fault model with the heterogeneous dynamic stress drops evaluated by the procedure in Fig. 7.4. We confirmed that the shortperiod level of the model in Fig. 7.7 was $10 \%$ larger than that of the model in Fig. 7.6.

Next, we calculated strong ground motions at 10-km-mesh points around the faults by the stochastic Green's function method [19].

Figure 7.8 compares the peak ground accelerations and velocities for the model with the uniform dynamic stress drops on the asperities and the ground motion prediction equations by $\mathrm{Si}$ and Midorikawa [4]. Figure 7.9 compares the peak ground accelerations and velocities for the model with heterogeneous dynamic stress drops and the ground motion prediction equations by $\mathrm{Si}$ and Midorikawa [4]. We find that the accelerations and velocities in Fig. 7.9 have larger deviation than those in Fig. 7.8. Especially, in the vicinity of the fault trace, while most of the peak ground accelerations and velocities for the model with the uniform dynamic stress drops are within the mean plus/minus standard deviation of the ground

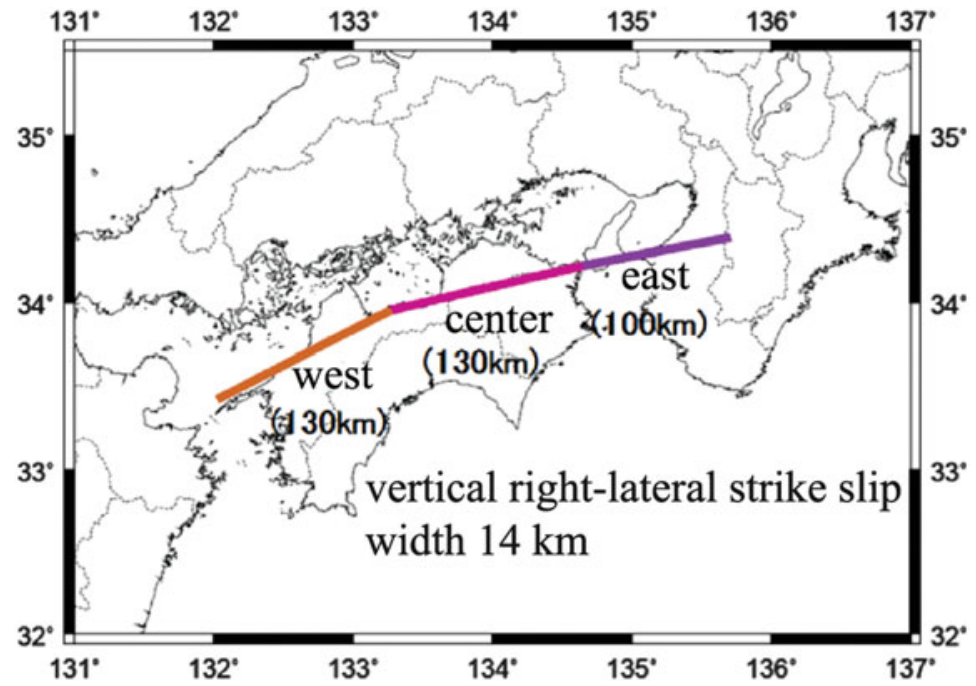

Fig. 7.5 Median Tectonic Line, Japan, and a fault model for strong motion prediction 
eastern fault $(100 \mathrm{~km})$

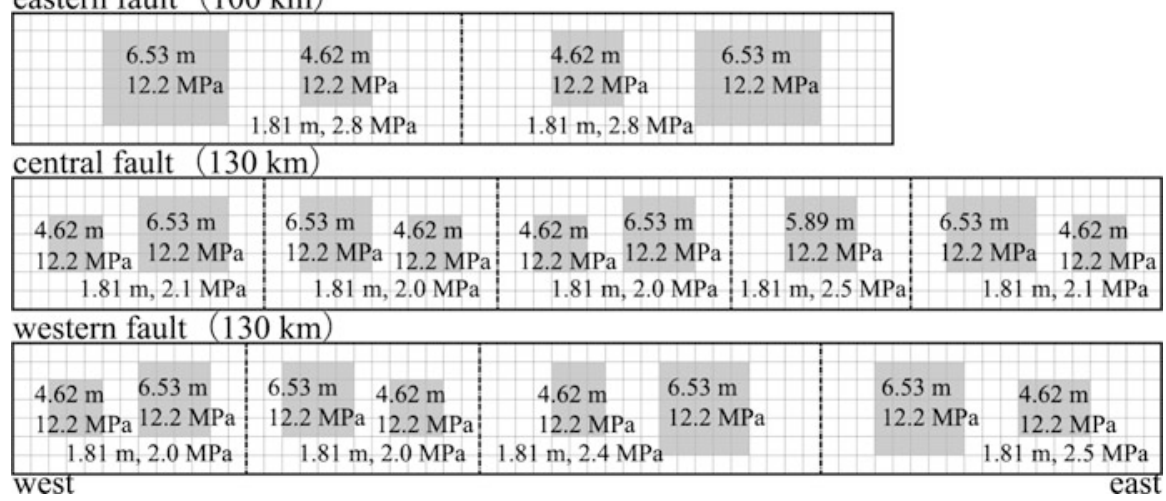

Fig. 7.6 Fault model with uniform dynamic stress drops on the asperities

eastern fault $(100 \mathrm{~km})$

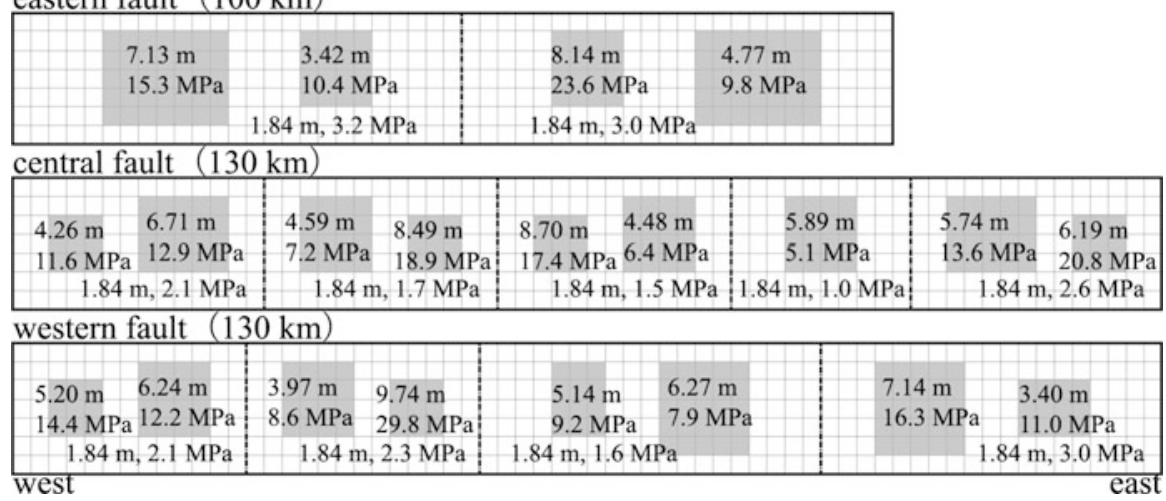

Fig. 7.7 Fault model with heterogeneous dynamic stress drops on the asperities

motion prediction equations by Si and Midorikawa [4], some of those for the model with the heterogeneous dynamic stress drops are beyond the mean plus standard deviation. However, the averages are almost the same. 

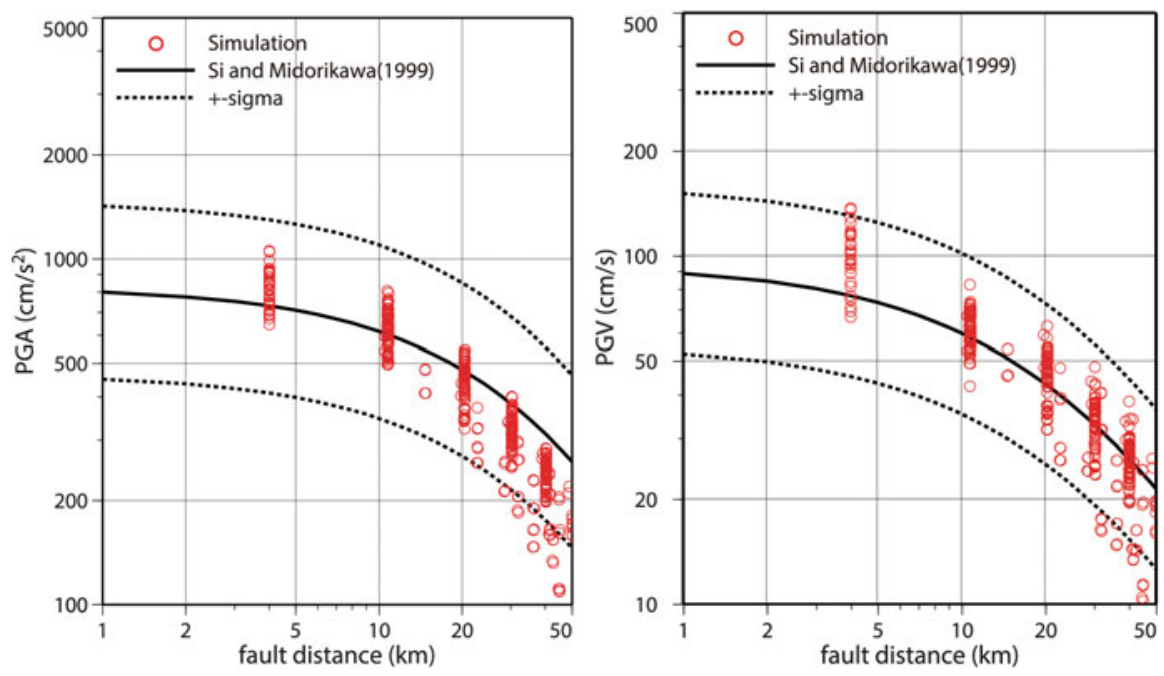

Fig. 7.8 Comparison between the strong motions predicted by the fault model with uniform dynamic stress drops on the asperities and ground motion prediction equations by $\mathrm{Si}$ and Midorikawa [4]
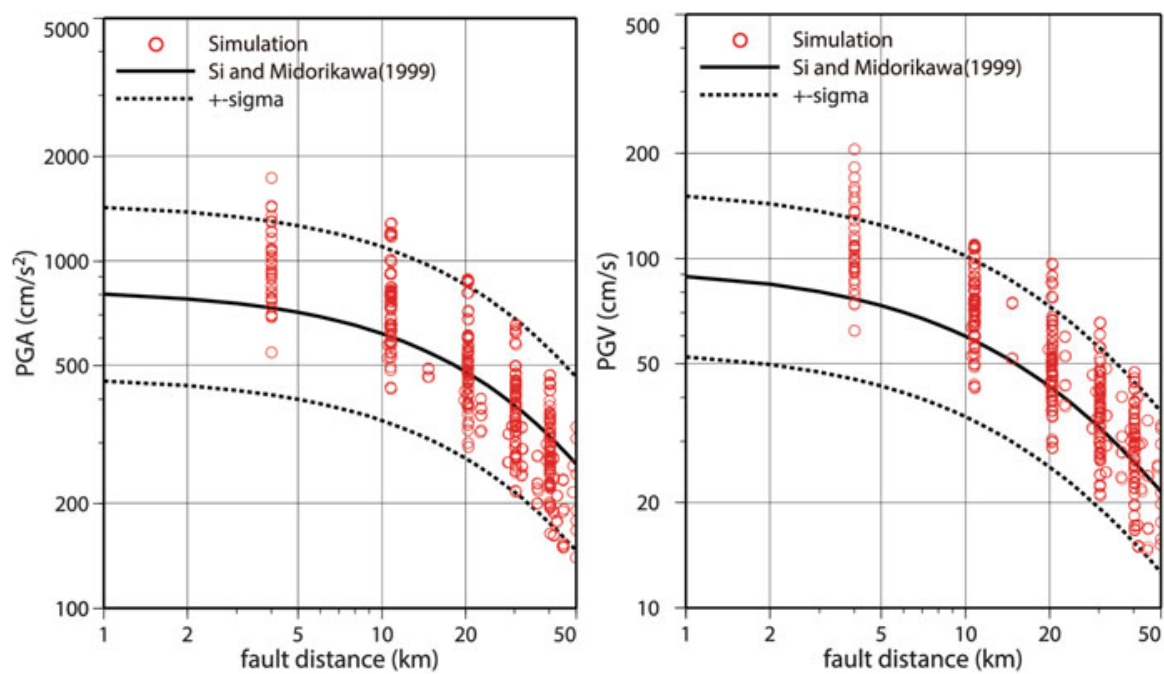

Fig. 7.9 Comparison between the strong motions predicted by the fault model with heterogeneous dynamic stress drops on the asperities and ground motion prediction equations by $\mathrm{Si}$ and Midorikawa [4] 


\subsection{Conclusions}

We proposed a procedure for evaluating fault parameters taking into account of the heterogeneous dynamic stress drops on the asperities and calculated strong ground motions. The results had wider variations of the peak ground accelerations and velocities than those with the uniform dynamic stress drops on the asperities, while the averages were almost the same.

The procedure proposed in this paper can be applied not only to very long faults of $\mathrm{M}_{\mathrm{W}} 8$ class earthquakes but also to medium-sized faults of $\mathrm{M}_{\mathrm{W}} 7$ class earthquakes.

Acknowledgments This study is a part of the examination results in Research Project on Seismic Hazard Assessment for Japan by the National Research Institute for Earth Science and Disaster Prevention.

Open Access This chapter is distributed under the terms of the Creative Commons Attribution Noncommercial License, which permits any noncommercial use, distribution, and reproduction in any medium, provided the original author(s) and source are credited.

\section{References}

1. Dan K, Ju D, Irie K, Arzpeima S, Ishii Y (2011) Estimation of averaged dynamic stress drops of inland earthquakes caused by long strike-slip faults and its application to asperity models for predicting strong ground motions. J Struct Constr Eng (Trans Archit Inst Japan) 76(670): 2041-2050

2. Dan K, Ju D, Shimazu N, Irie K (2012) Strong ground motions simulated by asperity models based on averaged dynamic stress drops on inland earthquakes caused by long strike-slip faults. J Struct Constr Eng (Trans Archit Inst Japan) 77(678):1257-1264

3. Dan K, Irie K, Ju D, Shimazu N, Torita H (2015) Procedure for estimating parameters of fault models of inland earthquakes caused by long reverse faults. J Struct Constr Eng (Trans Archit Inst Japan) 80(707):47-57

4. Si H, Midorikawa S (1999) New attenuation relationships for peak ground acceleration and velocity considering effects of fault type and site condition. J Struct Constr Eng (Trans Archit Inst Japan) (523):63-70

5. Kamae K, Irikura K (1997) A fault model of the 1995 Hyogo-Ken Nanbu earthquakes and simulation of strong ground motion in near-source area. J Struct Constr Eng (Trans Archit Inst Japan) (500):29-36

6. Kamae K, Irikura K (2002) Source characterization and strong motion simulation for the 1999 Kocaeli, Turkey and the 1999 Chi-Chi, Taiwan earthquakes. In: Proceedings of the 11th Japan earthquake engineering symposium, pp 545-550

7. Ikeda T, Kamae K, Miwa S, Irikura K (2002) Strong ground motion simulation of the 2000 Tottori-Ken Seibu earthquake using the hybrid technique. In: Proceedings of the 11th Japan earthquake engineering symposium, pp 579-582

8. Muto M, Shimazu N, Dan K, Abiru T (2009) Asperity models for the crustal earthquakes in Chugoku District based on short-period level by spectral inversion, Part 2: 2000 Tottori-Ken Seibu earthquake, Summaries of technical papers of Annual Meeting Architectural Institute of Japan, pp 151-152 
9. Satoh T, Kawase H (2006) Estimation of characteristic source model of the 2005 west off Fukuoka prefecture earthquake based on empirical Green's function method. In: Proceedings of the 12th Japan earthquake engineering symposium, pp 170-173

10. Kamae K, Ikeda T, Miwa S (2005) Source model composed of asperities for the $2004 \mathrm{Mid}$ Niigata Prefecture, Japan, earthquake $\left(\mathrm{M}_{\mathrm{JMA}}=6.8\right)$ by the forward modeling using the empirical Green's function method. Earth Planets Space 57:533-538

11. Satoh T, Hijikata K, Uetake T, Tokumitsu R, Dan K (2007) Cause of large peak ground acceleration of the 2004 Niigata-Ken Chuetsu earthquake by broadband source inversion, Part 2. Middle and short-period source inversion, Summaries of technical papers of Annual Meeting Architectural Institute of Japan, pp 365-366

12. Kamae K, Ikeda T, Miwa S (2014) http://www.rri.kyoto-u.ac.jp/jishin/eq/notohantou/ notohantou.html. Referred on 3 June 2014

13. Kurahashi S, Masaki K, Irikura K (2008) Source model of the 2007 Noto-Hanto earthquake (Mw 6.7) for estimating broad-band strong ground motion. Earth Planets Space 60:89-94

14. Irikura K, Kagawa T, Miyakoshi K, Kurahashi S (2014) http://www.kojiro-irikura.jp/pdf/ jishingakkai2009PPT.pdf. Referred on 3 June 2014

15. Kamae K, Kawabe H (2014) http://www.rri.kyoto-u.ac.jp/jishin/eq/niigata_chuetsuoki_5/ chuuetsuoki_20080307.pdf. Referred on 3 June 2014

16. Kamae K (2014) http://www.rri.kyoto-u.ac.jp/jishin/iwate_miyagi_1.html. Referred on 3 June 2014

17. Irikura K, Kurahashi S (2014) Modeling of source fault and generation of high-acceleration ground motion for the 2008 Iwate-Miyagi-Nairiku earthquake, Programme and Abstracts, Japanese Society of Active Fault Studies 2008 Fall Meeting. http://www.kojiro-irikura.jp/ pdf/katudansougakkai2008.pdf. Referred on 3 June 2014

18. Irikura K, Kurahashi S (2009) "Recipe" of strong motion prediction for great earthquakes with mega fault systems, Programme and Abstracts, The Seismological Society of Japan 2009, Fall Meeting. http://www.kojiro-irikura.jp/pdf/jishingakkai2009PPT.pdf. Referred on 3 June 2014

19. Dan K, Ju D, Muto M (2010) Modeling of subsurface fault for strong motion prediction inferred from short active fault observed on ground surface. J Struct Constr Eng (Trans Archit Inst Japan) 75(648):279-288 